\title{
Cracks Propagation as a Function of Grain Size Variants on Nanocrystalline Materials' Yield Stress Produced by Accumulative Roll-Bonding
}

\author{
P. B. Sob ${ }^{1 *}$, A. A. Alugongo ${ }^{1}$, T. B. Tengen ${ }^{2}$ \\ ${ }^{1}$ Departmentof Mechanical Engineering, Faculty of Engineering and Technology, Vaal University of Technology, Vanderbijlpark, \\ South Africa \\ ${ }^{2}$ Department of Industrial Engineering and Operations Management, Faculty of Engineering and Technology, Vaal University of \\ Technology, Vanderbijlpark, South Africa \\ Email: ‘baonhe_sob@rocketmail.com, alfayoa@vut.ac.za, thomas@vut.ac.za
}

How to cite this paper: Sob, P.B., Alugongo, A.A. and Tengen, T.B. (2017) Cracks Propagation as a Function of Grain Size Variants on Nanocrystalline Materials' Yield Stress Produced by Accumulative RollBonding. Advances in Materials Physics and Chemistry, 7, 58-69.

https://doi.org/10.4236/ampc.2017.72006

Received: December 22, 2016

Accepted: February 17, 2017

Published: February 20, 2017

Copyright $\odot 2017$ by authors and Scientific Research Publishing Inc. This work is licensed under the Creative Commons Attribution International License (CC BY 4.0).

http://creativecommons.org/licenses/by/4.0/

\begin{abstract}
Cracks are usually observed at the edge of materials deformed by accumulative roll bonding from conventional materials to nanostructure materials. The observed cracks then propagate in the materials during grain refinement. The cracks propagation affects the yield stress and the effective fracture energy of nanocrystalline materials. In this study, the impacts of crack propagation when measured as a function of grain size variants on nanocrystalline materials' yield stress are investigated for a material deformed by accumulative roll-bonding. The study employs experimental data and theoretical concepts of severe plastic deformation and cracks processes in nanocrystalline materials. The current studies also focus on nano-cracks that will not lead to rapid materials failure during grain refinement. The study revealed that crack propagation varied as a function of grain size variants during grain refinement. The study also revealed that nano-crack increased during the deformation of nanostructured materials. The study also revealed that the effective fracture energy decreased as grain refinement took place. The study revealed that nanomaterials yield stress decreased with the increase in effective fracture energy. The current study suggests a theoretical model that shows the generation of nanomaterials cracks during grain refinement as a function of grain size variants. In the model, the cracks propagate on nanocrystalline materials due to the compressive load applied to a material. The model predicts that the generation of cracks as functions of grain size variants impacts the energy level in nanocrystalline materials.
\end{abstract}

\section{Keywords}

Nanostructure, Grain Size Variants, Stress, Crack, Energy 


\section{Introduction}

Nanocrystalline (nc) materials exhibit high strength but with low tensile ductility and low fracture toughness that limits their applications [1]-[6]. The main reasons why material properties are studied are to prevent material failure during usage [7]. A crack in a material is an indication that the produced material might eventually fail during usagesince cracks which have negative impacts on nc materials mechanical properties [7]. However, some cracks do not always lead to material failure and as such there are different classifications of cracks propagation [8].

The first category of crack propagation is that which the produced nanomaterials' is subjected to high risk of failure during usage. The initiated cracks and subsequent cracks propagation occurred quite readily due to lacks of microstructural barriers that could prevent the crack propagation [8]. This is mostly seen in glass and conventional materials [8]. Cracks that propagate slowly are nanocracks because their initiation and subsequent propagation are prevented due to high curvature grain boundaries which normally prevent their propagation [9]. This is mostly found in nanocrystalline materials produced through the top down approach of manufacturing [9].

Material crack propagation must be studied to prevent materials failure during usage for materials that can fail. Nano-crack propagation must be studied to understand the impact of nano-cracks propagation on nc materials during grain refinement. There is therefore, no doubt that over the past years, several researchers have succeeded in studying crack propagation on nc materials during grain refinement. Most of their studies focused on cracks that led to materials failure [1]-[8]. Few researchers focused on nano-cracks that would not lead materials failures. In fact, most of their studies have shown how generated cracks affect the mechanical properties of nc materials during grain refinement. Few researchers focused on nano-cracks that did not lead materials immediate failures. In fact, most of their studies have shown how generated cracks affect the mechanical properties of nc materials during grain refinement; for example nanomaterials cracks have a negative impact on the strength and energy (i.e. on the load that the material can withstand) during grain refinement.

Some researchers have proposed theoretical models to study crack propagation on nc materials. In some of their studies, the relationship between rotational deformation and grain size was studied [1]-[8]. It must be noted that those mentioned previous studies on nanomaterials crack propagation do not take into consideration the different ways in which grain size can be measured during grain refinement in nanomaterials, with the different ways of measuring grain size hereby called grain size variants of nc materials [1]-[11]. Nanocrystalline materials are generally known for their high surface-to-volume ratios of their grains that tend to affect their properties [10], with some investigation success having been achieved by microscopy analysis [11] [12].

The high surface-to-volume ratios of nanocrystalline materials' grains greatly depend on the size function of nanomaterials' grains during manufacturing [10]. 
Most often the high surface-to-volume ratios have not been properly modeled theoretically since most, existing models that relating nanocrystalline materials properties and grain size have been derived while considering only the equivalent radius. Thus, most theoretical modeled results cannot give proper correlation with microscopy results since most theoretical models dealt only with the equivalent radius [9].

The transmission electron microscopy (TEM) and most microscopy indicate that there are different approaches of measuring grain size (i.e. grain size variants) which give different parameters which most developed models that dealt only with the equivalent radius ignored [11] [12]. A grain deformed by severe plastic deformation (SPD), specifically the accumulative roll-bonding (ARB) and equal channel angular pressing (ECAP), may be observed from three different directions [11] [12] which are the direction of applied load known as the normal direction (ND), transverse direction (TD) and the rolling direction (RD) or sliding direction (SD) as shown in Figures 1(a)-(c).

The use of the equivalent radius only cannot give more information for example about the shape of a grain during grain refinement. Since different approaches of measuring grain size exist, the crack on nc materials propagates in different ways when measured as function of the material grain size variants. Thus the materials grain size variants experience different degree of cracks propagation during grain refinement. It is important to develop a theoretical model that shows the generation of nanomaterials crack as function of grain size variant. From the theoretical developed models other factors of nc materials stability and energy can be investigated. From most revealed microscopic results, nano-cracks are normally observed without proper information of crack growth as a function of TD, ND and RD.

Therefore, nano-cracks propagation has been previously analyzed without taking into consideration the different direction along which grain size can be measured given by the TD, ND and RD [11] [12]. It clear that, it is difficult to correlate theoretical results and experimental findings. It is also important to study nano-cracks on microstructures that will not subject nanomaterials' to any risk of failure. This will also help researchers to understand the critical grain size in which cracks propagation can be prevented to avoid materials failure during manufacturing. In this current research finding, the result of our theoretical

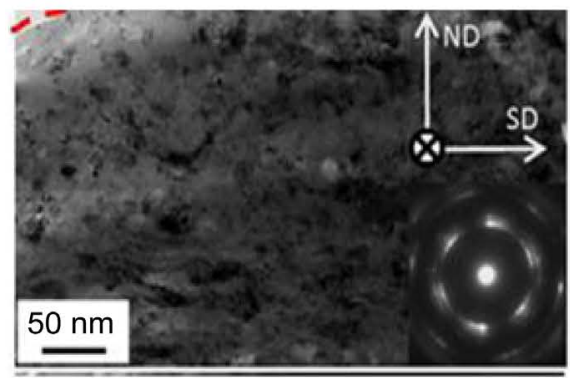

(a)

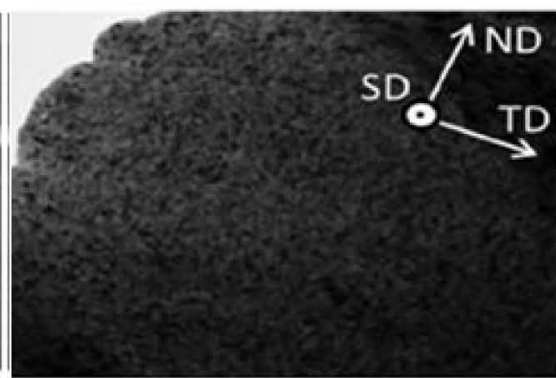

(b)

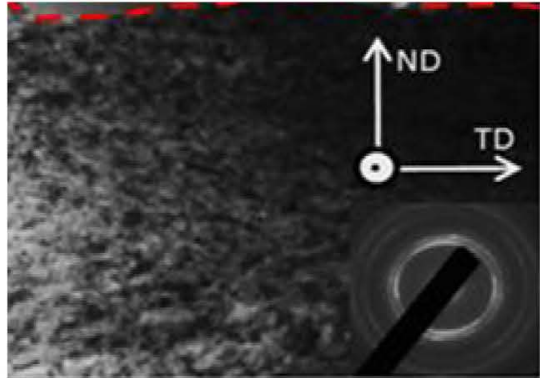

(c)

Figure 1. (a)-(c) dark-field TEM microstructures and texture evolution revealing different direction of measuring grain size i.e. $\mathrm{TD}, \mathrm{ND}$ and RD or SD [11] [12]. 
modeling and simulation will guide nanoscientists, nanotechnologists, nanoengineers and materials scientists during production of nanomaterials by ARB.

The objective of this paper is to study the effect of crack propagation as function of grain size variants on nc materials during grain refinement by ARB. In this paper we focus on experimental data, theoretical concepts of severe plastic deformation and cracks processes on nc materials. Our study also focuses on modeling nano-cracks that do not lead to material failure during severe plastic deformation by ARB. The modeled results revealed how cracks propagation, effective fracture energy and grain size variants vary manufacturing of nanomaterials by ARB. The reason why we focused on the ARB method is based on the fact that most industries have adopted the ARB since it improves on productivity [9].

\section{Methodology}

\subsection{Theoretical Framework and Consideration for Model Derivation}

For proper modeling of crack propagations the following factors must be taken into consideration. The conditions in which the crack was initiated since we cannot talk of crack propagation without looking at crack initiation. In this project crack initiation occurred due to material fatigue after several ARB cycles since it was necessary to performed several ARB passes for conventional materials to become nanomaterials. From the study crack propagation occurs from existing initiated cracks due to material fatigue during excessive ARB cycles. It is important to look at the method of materials manufacturing due to the fact that crack propagation depend on the nature of forces or load acting on materials during deformation. Therefore, the choice of models equation will also depend on forces, load and the nature of materials deformation.

It is also important to understand how the grain sizes induce the change of crack propagation on the grain size variants during grain refinement. During the grain refinement process, different grain curvatures are generated on the grain size variants. The different grain curvatures generated are due to different material flow along the TD, RD and ND. Since different materials flows are observed along the $\mathrm{ND}, \mathrm{TD}$ and $\mathrm{RD}$, the material flow is higher along the elongated directions RD and TD when compared with ND [9]. Since more materials flow occurred at the $\mathrm{RD}$ and $\mathrm{TD}$, more grain dislocations; grain curvatures, grain boundaries migration and grain rotation coalescence event took place along the $\mathrm{RD}$ and TD when compared to ND.

Since more grain dislocation, grain boundaries migration and grain rotation coalescence activities took place in the elongated directions more grain curvatures are generated in the $\mathrm{RD}$ and $\mathrm{TD}$ [9]. Two grain curvatures exists during grain refinement, the high angle grain boundaries curvatures that gives enhanced properties which hinder crack propagation during grain refinement and low angle grain curvatures boundaries that give lower properties which enable crack propagation during grain refinement [9]. Therefore, the grain sizes in- 
duced the change of crack Propagation due different grain size curvatures on the grain boundaries.

\subsection{Method of Manufacturing Nanomaterials}

The experimental set up for ARB is shown in Figure 2(a). The ARB technology uses conventional rolling facility. For this project two shafts were placed horizontally so that they were free to rotate by means of a mechanically automated operation. During experimentation the sample was forced or fed through the rotating shafts. The rotating shafts gripped the sample and forced the sample through the rollers in the first pass. The deformed sample was cut in two pieces and stacked together. Before stacking, the entire surfaces of the strips were wirebrushed (i.e. using stainless steel brush) and degreased with tetrachlorethylene to achieve good bonding. The materials were joined together in the corners using aluminum wires and subsequently rolled. The whole sequence of "rolling, cutting, face-brushing, degreasing and stacking" was repeated again for several "passes" since it is necessary to performed several passes by ARB for conventional materials to become nanomaterials.

During materials deformation by ARB the material was subjected to compressive cyclic loading condition as shown in Figure 2(d). Figure 2(c) revealed an image of cracks captured by scanning electron microscopy (SEM) during the deformation of nanostructure materials. Figure 2(b) shows the different approaches of measuring grain sizes which are along the TD, ND and $\mathrm{RD}$ as revealed

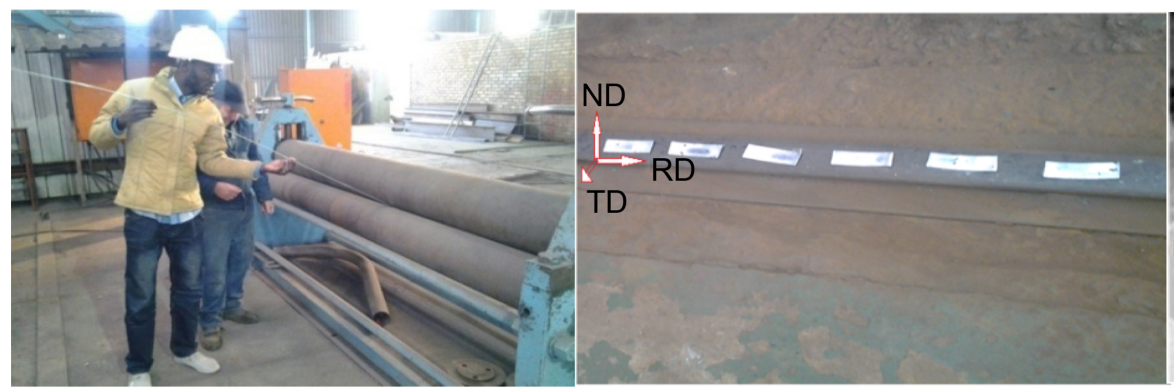

(a) (b)

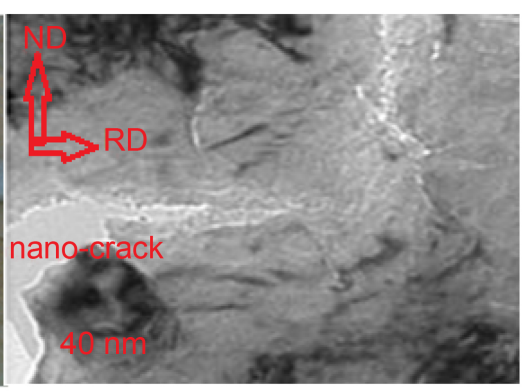

(c)

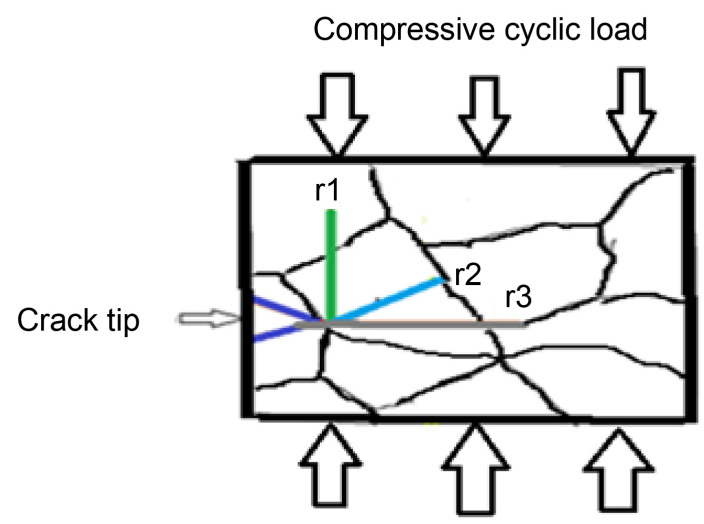

(d)

Figure 2. (a) ARB process (b) deformed materials after ARB cycles (c) SEM Image of nc material cracks (d) schematic diagram of compressive cyclic loads. 
by [11] [12]. In this research project, the measurement of grain size along the TD during experimentation is represented in the model as the semi major axis length $r_{1}$, the ND during experimentation is represented in the model as the semi minor axis length $r_{2}$, and the $\mathrm{RD}$ or SD during experimentation is represented in the model as the major axis length $r_{3}$ and the equivalent radius $r$ is defined as the radius of an equivalent sphere or circle obtained by the displacement method of volume/area measurement.

\subsection{Theoretical Consideration: Choice of Model Equations and Justification}

The griffith crack theory conditions from empirical data states [7], the Griffith crack theory is used for homogeneous continuum materials. The Griffith crack size can greatly exceed the nanomaterials' grain size of (approximately $10 \mathrm{~nm}$ ) due to high density of their GBs and their junctions. In the developed models of the grain size variants in this research, GBs, GRC, rate of grain breakage etc. are some of the major mechanism of grain size evolution that are considered also in the Griffith Models. We are aware that high angle grain boundaries in nanomaterials enhanced their materials properties and a low angle boundary doesn't enhance their properties. It is therefore important to study how crack propagation affects material properties using the Griffith crack size. The induced crack size at the edge of a deformed materials can be represented by the Griffiths crack size [7] given by

$$
L_{G}=\frac{4 E \gamma_{0}^{*}}{\pi\left(1-v^{2}\right) \sigma_{f}^{2}} \text { at } \gamma_{0}^{*}=\frac{\mu b}{20}
$$

where $b$ is the interatomic distance, $v$ is the Poisson ratio, $\sigma_{f}$ is the nanocracking stress given as $\sigma_{f}=\frac{\mu}{50}, \mu$ is the shear modulus, $\gamma_{0}^{*}$ is the crack effective fracture energy in nc materials and $E$ is the young's modulus [9]. The expression of young's modulus $E$ of nanomaterials is obtained from [9]

$$
E=\left(\frac{P}{d}\right)\left(\frac{L^{3}}{192 I}\right)
$$

where the moment of initial is $\mathrm{I}=\pi D / 64, D=r_{2}$ is the radius of the grain as measured in the normal direction, $L=r_{1}$ is the radius in the transverse direction, $d=\mathrm{d} r=\left(r-r_{0}\right)$ is the change of equivalent grain size during grain refinement where $r$ is the variable equivalent grain size, $r_{0}$ is the fixed grain size and $P$ is the applied force during grain deformation [9]. Simplifying expression (2) gives

$$
E=\left(\frac{P}{3 \pi}\right)\left(\frac{r_{1}^{3}}{r_{2}^{4}}\right) \frac{1}{\left(r-r_{0}\right)} .
$$

The condition of initiation of trans and intercrystallite fracture are given by the relation between the cohesion $\left(\gamma_{0}\right)$ and grain boundary fracture energies $\left(\gamma_{e}\right)[7]$ given as 


$$
\gamma_{0}=2 \gamma, \gamma_{e}=\eta\left(2 \gamma+2 \gamma_{s}-\gamma_{b}\right)
$$

where $\gamma$ and $\gamma_{b}$ are the specific energies of the free surface and grain boundary (GB), respectively, $\gamma_{s}$ is the cleavage step energy; and $\eta$ is the roughness factor of the fracture surface. In polycrystalline materials, the contribution of GB junction to the fracture energy is very negligible and is ignored during fracture analysis [7]. The volume fraction in a material consists of GBs and triple junctions and it increases as the grain size decreases. For crack propagation in nc materials, the effective fracture energy is given as

$$
\gamma_{0}^{*}=f_{0} \gamma_{0}+f_{b} \gamma_{e}+f_{j} \gamma_{j}
$$

where $f_{0}, f_{b}$, and $f_{j}$ are the crack area/volume fractions in the internal volume of grains, GBs, and GB junctions, respectively, which depend on the crack propagation trajectory, and $\gamma_{0}, \gamma_{b}$, and $\gamma_{j}$ are the contributions of the corresponding structural components to the specific energy of the nc material fracture. At any instance during grain refinement the crack area/volume fractions of $f_{0}, f_{b}$, and $f_{j}$ can be related to the equivalent volume of the grain.

The characteristic values of material parameters such as $\gamma_{0}, \gamma_{b}$, and $\gamma_{j}$ are the corresponding structural components to the specific energy of the nc material fracture. The energy of large-angle GB is approximately three times lower than the free-surface energy $\gamma_{b} \approx \frac{\gamma}{3}$ [7]. The energy of nonequilibrium boundaries with excess dislocation density $\rho$ is $\gamma_{b n} \approx \frac{4}{3} \gamma$, so it gives $\gamma_{e} \approx \frac{u b}{30}$ [7]. Since the induced material crack propagates on grain size variants during grain refinement, the next step is to employ the different models of grain size variants during grain refinement from which the effects of crack propagation are studied. The models of nc materials grain size variants as derived by [9] are given as

$$
\mathrm{d} r_{1}=M\left(\frac{1}{r_{c 1}}+\frac{1}{r_{1}}\right) \mathrm{d} t+r_{1}^{\frac{1}{2}} D \mathrm{~d} W(t)-Z r_{1} V_{1} \mathrm{~d}(t)
$$

where $r_{c 1}=$ local critical grain size, $Z$ and $D$ are Constants, $\mathrm{d} W(t)=$ change of the Wiener process, $V_{1}=\tau_{1} r_{1}^{2}$ defines rate of grain breakage,

$$
\begin{gathered}
M=M_{0}\left(1+\frac{C D}{r_{1}}\right), C D=4(H m)\left(h_{0}\right) /((k)(t)), T_{m}=T\left\{\ln \left(m_{01} / m\right)\right\} \text { and } \\
M_{0}=M_{01} \exp \left\{-T_{m}(\inf ) / T\right\} . \\
\mathrm{dr}_{3}=\operatorname{Ratio}_{1}\left(\mathrm{~d} r_{1}\right) \\
\mathrm{d} r=-O r d t+I \mathrm{~d} W(t)
\end{gathered}
$$

where $O$ and $I$ are constants.

$$
\mathrm{d} r_{2}=\operatorname{Ratio}_{2}(\mathrm{~d} r) .
$$

The yield stress on nanomaterial's grain subjected to plastic deformation is given by Zhao [13]

$$
\sigma(r)=\sigma_{0}^{\prime}+A\left(r^{-\frac{1}{2}}\right)-B\left(r^{-1}\right)+C\left(r^{-\frac{3}{2}}\right)
$$


where $\sigma_{0}^{\prime}=\sigma_{0}+K_{t}$ is bulk yield stress, $A=K_{d}$ is HPR proportionality constant, $B=K_{t}\left[2 h H_{M} / R T_{r}\right], C=K_{d}\left[2 h H_{M} / R T_{r}\right], K_{t}$ is a constant, $h$ is atomic diameter in the case of metal, $H_{m}$ is the bulk melting enthalpy, $R$ is ideal gas constant, $T_{r}$ is the room temperature, $K_{d}>100 K_{t}$ and $\sigma_{0}>10 K_{t}$. Equations (1) to (10) are stochastic and are solved simultaneously using lognormal distribution of grain size.

\section{Results and Discussion}

To test the models proposed in this report, the data from (nanocrystalline) aluminum sample (some of which are found in other reports: [9] [14]) are used, which are $M_{0}^{\prime}=0.01 \mathrm{~nm}^{2} \cdot \mathrm{s}^{-1}, \quad m=4, r_{c 1}=1.95 r, \quad C C=12, a=0.90$, $D=10^{-4}, \quad h_{0}=0.25 \mathrm{~nm}, T_{m}(\infty)=933.47 \mathrm{~K}, \quad C V_{0}=0.3$, $H_{m}(\infty)=10.71 \mathrm{KJ} \cdot \mathrm{Mol}^{1}, \quad \sigma_{0}^{\prime}=16.7 \mathrm{MPa}, \quad K_{t}=1.3, \quad \sigma_{0}=15.40 \mathrm{MPa}$, $K d=1301.77 \mathrm{MPa} \_\mathrm{nm}{ }^{1 / 2}, \quad R=8.31 \mathrm{JK}^{-1} \cdot \mathrm{mol}^{-1}$ and $T_{r}=300 \mathrm{~K}$, data from [15] [16] [17] are also used, which are $v=0.34, \mu=0.129 \times 10^{10} \mathrm{~Pa}$, $\gamma=0.98 \pm 0.21 \mathrm{~J} \cdot \mathrm{m}^{-1}$. The additional data obtained for this work are $O=0.0035, \quad I=1.1, \quad r_{0}=100 \mathrm{~nm}, Z=0.4$, Ratio $_{1}=0.81$, Ratio $_{2}=1.071$, and $\tau_{1}=0.000008$. The additional data were obtained through curve fitting of the empirical data from the different measures of the sizes. The obtained results are presented in the plots below.

From Figure 3(a) it is observed that the material crack increases during grain

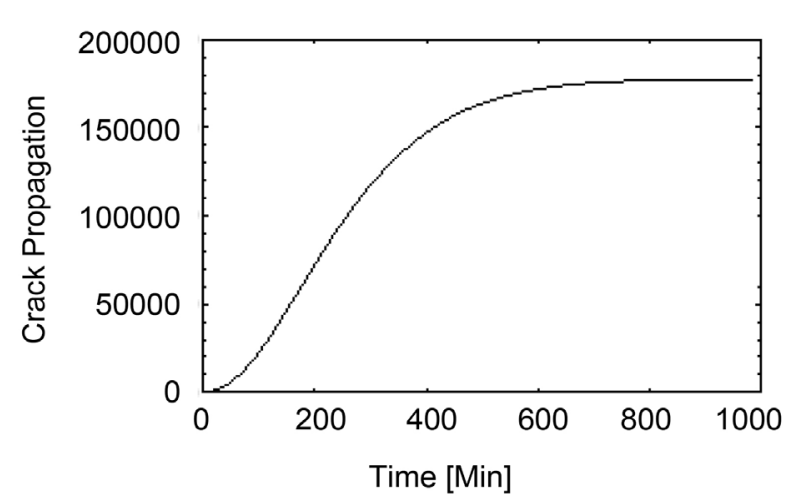

(a)

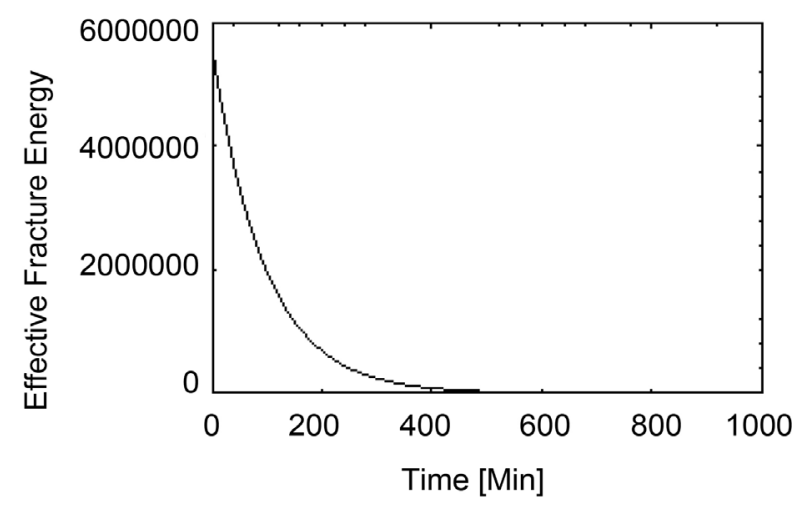

(b)

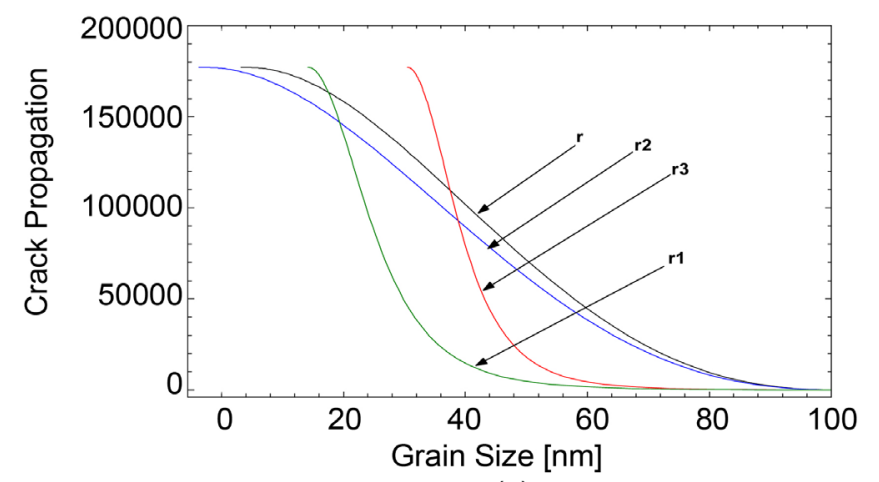

(c)

Figure 3. (a): Crack Propagation as a Function of Time [S] (b) Effective Fracture Energy as a Function of Time [S] and (c) Crack Propagation as a Function of Size [nm]. 
refinement of nc materials. It is also observed from Figure 3(b) that the effective fracture energy of nc materials decreases during grain refinement. Figure 3(a) and Figure 3(b) also indicates that during the deformation of nc materials the crack propagation decreases the effective fracture energy when the material grain size decreases. This can be explained based on the fact that during grain refinement more dislocations are usually generated at the crack tip section of the material, as it is seen that crack propagation affect the effective fracture energy of $\mathrm{nc}$ materials during grain refinement. Figure $3(\mathrm{c})$ revealed that as the material grain size decreases the propagation of material crack increases on the grain size variants during grain refinement of nc materials.

It is also observed from Figure 3(c) that as the material grain size decreases the increase of crack propagation on the grain size variant differs. It is observed from Figure 3(c) that the materials crack propagation is higher when measured along $r$ followed by $r_{2}$ and $r_{3}$ while the material crack propagation is lower along $r_{1}$. This can be explained based on the fact that during grain refinement the material experience more grain dislocation in the section of the crack tip along $r, r_{2}$ and $r_{3}$ and less grain dislocation along $r_{1}$. During the initial process of grain refinement the material crack propagation is higher along $\mathrm{r}$ and $r_{2}$ due to lower angle grain boundaries (GBs) and lower along $r_{1}$ and $r_{3}$ due to higher angle GBs. As more grain refinement took place the crack propagation increase along $r_{1}$ and $r_{3}$ since the angles of their GBs become lower when compared to $r$ and $r_{2}$. It is observed that the cracks propagation on nc material depend on the GBs described by the grain size variants since materials with higher GBs experience lower crack propagation and materials with lower GBs experience higher crack propagation during grain refinement.

The opening of crack in a material is always driven by local stress concentration [15] [16] [17] [18] [19]. The stress in the material is usually higher near the tips of the crack in the material. Since the stress in the material is usually higher near the crack tips in the material, the material crack propagates along the GBs of the materials. It is also noticed that at larger grain size the material atoms resist fracture, as the material has a stronger fracture resistance on the material grain size variants. The obtained result in this study is in line with that reported by other researcher [15] [16] [17] [18] [19].

As explained earlier in Figure 4(a) and Figure 4(b) crack propagation increases with time while the effective fracture energy decreases during grain refinement. This is an indication that during the deformation of nc materials, crack propagation decreases the effective fracture energy. It is observed from Figure 4(a) that the effect fracture energy is high for larger grain size variants and low for smaller grain size variants. It is also observed from Figure 4(a) that at larger grain size and smaller grain size the effective fracture energy varies with the grain size variants. The effective fracture energy is higher for a larger grain size of $r_{1}$ followed by $r_{3}$ and lower for $r_{2}$ and $\mathrm{r}$ due to different dislocation activities as function of the material grain size variants during crack propagation.

The effective fracture energy in a material is related with the deformation 


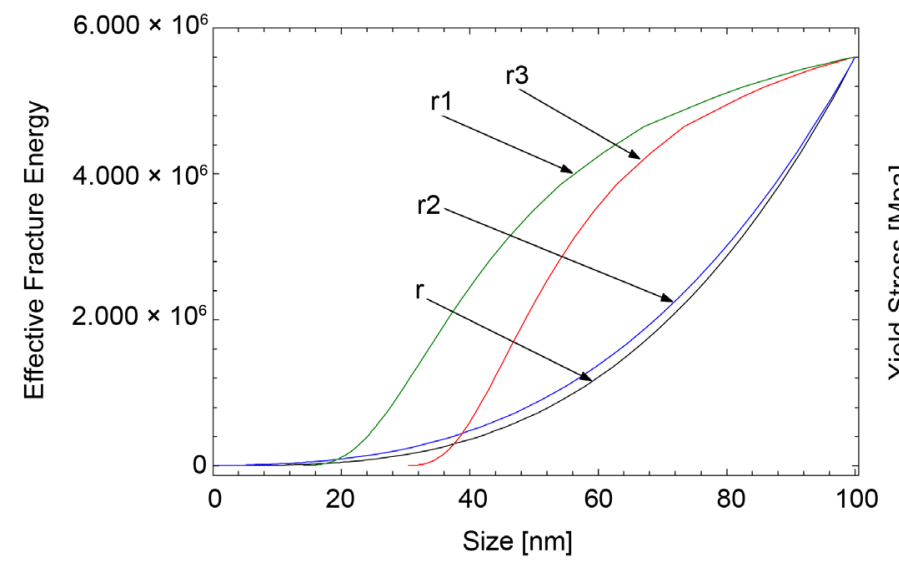

(a)

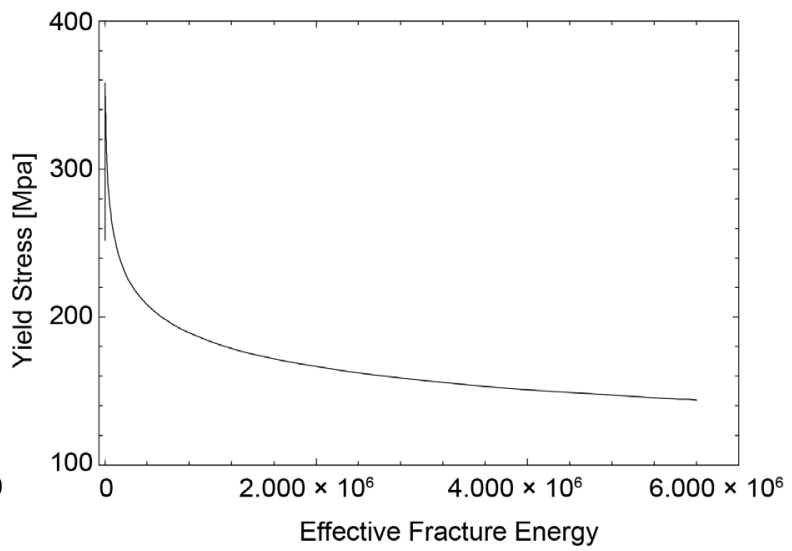

(b)

Figure 4. (a): Effective fracture energy evolution versus size [nm] (b) Yield stress [MPa] versus effective fracture energy.

mechanisms in the fracture process. It is clear as revealed in Figure 4(a) that the effective fracture energy is stronger for larger grain size, so they have higher resistance to crack propagation during grain refinement. As also revealed in Figure 4(a) the effective fracture energy is lower (weaker) for smaller grain size so they have lower resistance to crack propagation. Although larger grain size is important for ductility, its effects on materials strength and fracture toughness are uncertain. It is also observed from Figure 4(b) that the material yield stress decrease with increase in effective fracture energy during grain refinement. Thus it is shown that material crack propagation has a negative impact in nc material property during grain refinement.

\section{Conclusions}

The current work was aimed at studying the effect of crack propagation as a function of the grain size variants on nc materials during grain refinement by ARB. To achieve our objective, the model of Griffiths crack size was modified to be applicable to 3-D grain. The stochastic natures of the grain size variants have been also taken into consideration since the internal constituent structures of nanomaterials were random in nature and can be best studied using random processes specifically as stochastic processes. It can be concluded that crack propagation increases while the effective fracture energy decreases during deformation of conventional material to nanostructure materials. It is also observed that as the material grain size decreases, the increase in crack propagation differs for the grain size variants. It is also observed that at larger grain size, the material atoms resist fracture, since the fracture resistance in the material is stronger. It is however observed that at larger and smaller grain size, the effective fracture energy and crack propagation varies on the grain size variants. The effective fracture energy is higher for a larger grain size variant and the crack propagation is higher for smaller grain size.

The current research finding is of great significance since it has revealed results on cracks propagation that has never been revealed. Most previous findings 
of cracks propagation did not show in details how cracks propagation occurred on different directions of measurement of microstructures. If correlations were to be done on most previous research findings on nano-cracks propagation, there would be several controversies since most results of cracks propagation were not well classified according to the RD, TD and ND. Nanomaterials are generally known for their high surface-to-volume ratio which affects their properties. The high surface-to-volume ratio has not been well incorporated in most previous findings of nanomaterials physical and mechanical properties. Microscopy results give the surface area/volume but most existing theoretical models on nanomaterials physical and mechanical properties do not actually consider the surface area/volume on modeling and simulation which this research has considered.

In this current study, cracks propagation as a function of grain size variants gives useful results that can be correlated with microscopy results. For over the past decades, several results have been obtained with several controversies since the mechanism of size was never been clearly defined. In this research finding, the mechanism of size was defined based on the high surface-to-volume ratio which was one of the most important factors of nanomaterials. We can therefore, conclude that, our theoretical model that shows the generation of cracks on nc material as a function of size variants is practical for solids with grain size variants below a hundred nanometers. Since we have studied nano-cracks as a function of grain size variants that will not lead to materials failure during grain refinement, it will also be proper to study nano-cracks as function of grain size variants that will lead to material failure during grain refinement which is in progress by the same authors.

\section{Acknowledgements}

This material is based on the work which is supported financially by the National Research Foundation (NRF) and Vaal University of Technology (VUT).

\section{References}

[1] Meyer, M.A., Mishra, A. and Benson, D.J. (2006) Mechanical Properties of Nanocrystalline Materials. Progress in Materials Science, 51, 427-556. https://doi.org/10.1016/j.pmatsci.2005.08.003

[2] Kumar, K.S., Suresh, S. and Van Swygenhoven, H. (2003) Mechanical Behavior of Nanocrystalline Metals and Alloys. Acta Materialia, 51, 5743-5774. https://doi.org/10.1016/j.actamat.2003.08.032

[3] Koch, C.C. (2007) Structural Nanocrystalline Materials: An Overview. Journal of Materials Science, 42, 1403-1414. https://doi.org/10.1007/s10853-006-0609-3

[4] Dao, M., Lu, L., Asaro, R.J., Hosson, J.T. and Ma, E. (2007) Toward a Quantitative Understanding of Mechanical Behavior of Nanocrystalline Metals. Acta Materialia, 55, 4041-4065. https://doi.org/10.1016/j.actamat.2007.01.038

[5] Jiang, B. and Weng, G.J. (2004) A Generalized Self-Consistent Polycrystal Model for the Yield Strength of Nanocrystalline Materials. Journal of the Mechanics and Physics of Solids, 52, 1125-1149. https://doi.org/10.1016/j.jmps.2003.09.002

[6] Ovid'Ko, I.A. (2007) Review on the Fracture Processes in Nanocrystalline Materials. 
Journal of Materials Science, 42, 1694-1708. https://doi.org/10.1007/s10853-006-0968-9

[7] Pozdnyakov, V.A. and Glezer, A.M. (2005) Structural Mechanisms of Fracture of Nanocrystalline Materials. Physics of the Solid State, 47, 817-824. https://doi.org/10.1134/1.1924838

[8] Gilbert, C.J., Schroeder, V. and Ritchie, R.O. (1999) Mechanisms for Fracture and Fatigue-Crack Propagation in a Bulk Metallic Glass. Metallurgical and Materials Transactions A, 30A, 1739-1753. https://doi.org/10.1007/s11661-999-0173-y

[9] Sob, P.B., Alugongo, A.A. and Tengen, T.B. (2016) Modelling Strain Rate Sensitive Nanomaterials Mechanical Properties: The Effect of Varying Definitions. M. Tech. Dissertation, Vaal University of Technology, Vanderbijlpark.

[10] Ali Mansoori, G. (2004) Principles of Nanotechnology: Molecular-Based Study of Condensed Matter in Small Systems. World Scientific Publishing, Singapore.

[11] Hidalgo, P.M., Cepeda, J.C.M., Ruano, O.A. and Carreno, R.O.A. (2012) Effect of Warm Accumulative Roll Bonding on the Evolution of Microstructure, Texture and Creep Properties in the 7075 Aluminium Alloy. Materials Science and Engineering $A$, 556, 287-294. https://doi.org/10.1016/j.msea.2012.06.089

[12] Cai, W. and Bellon, P. (2013) Subsurface Microstructure Evolution and Deformation Mechanism of Ag-Cu Eutectic Alloy after Dry Sliding Wear. Wear, 303 602610. https://doi.org/10.1016/j.wear.2013.04.006

[13] Zhao, M. and Jiang, Q. (2006) Reverse Hall-Petch Relationship of Metals in Nanometer Size. IEEE Conference on Emerging Technologies-Nanoelectronics, Singapore, 10-13 January 2006, 472-474.

[14] Tengen, T.B. (2008) Analysis of Characteristic of Random Microstructures of Nanomaterials. PhD Thesis, Witwatersrand.

[15] Hui, F., Zhang, L.C. and Liu, Y.W. (2013) Special Rotational Deformation and Grain Size Effect on Fracture Toughness of Nanocrystalline Materials. International Journal of Plasticity, 42, 50-64. https://doi.org/10.1016/j.ijplas.2012.09.015

[16] Yungguang, L. and Shen, T.D. (2013) Effect of Nano-Metal Particles on the Fracture Toughness of Metal-Ceramic Composite. Material and Design, 45, 67-71. https://doi.org/10.1016/j.matdes.2012.08.065

[17] Stephane, C., Christian, F., Demoustier-Champagne, S. and Bernard, N. (2004) Surface Tension Effect on the Mechanical Properties of Nanomaterials Measured by Atomic Force Microscopy. Physical Review B, 69, Article ID: 165410.

[18] Ovid'Ko, I.A., Sheinerman, A.G. and Aifantis, E.C. (2011) Effect of Cooperative Grain Boundary Sliding and Migration on Crack Growth in Nanocrystalline Solids. Acta Materialia, 59, 5023-5031. https://doi.org/10.1016/j.actamat.2011.04.056

[19] Ovid'Ko, I.A. and Sheinerman, A.G. (2012) Nanoscale Rotational Deformation near Crack Tips in Nanocrystalline Solids. Journal of Physics D: Applied Physics, 45, Article ID: 335301. https://doi.org/10.1088/0022-3727/45/33/335301 
Submit or recommend next manuscript to SCIRP and we will provide best service for you:

Accepting pre-submission inquiries through Email, Facebook, LinkedIn, Twitter, etc. A wide selection of journals (inclusive of 9 subjects, more than 200 journals)

Providing 24-hour high-quality service

User-friendly online submission system

Fair and swift peer-review system

Efficient typesetting and proofreading procedure

Display of the result of downloads and visits, as well as the number of cited articles Maximum dissemination of your research work

Submit your manuscript at: http://papersubmission.scirp.org/

Or contact ampc@scirp.org 